\title{
QoS Mapping for LTE Backhauling over OFDMA-PONs
}

\author{
Wansu Lim, Milos Milosavljevic, Pandelis Kourtessis, Member, IEEE, John M. Senior \\ Optical Networks Group, Science and Technology Research Institute (STRI), University of Hertfordshire \\ College Lane Campus, Hatfield, AL10 9AB, UK \\ Tel: +44 (0) 1707286279,e-mail:w.lim9@herts.ac.uk
}

\begin{abstract}
This paper reports on the quality of service (QoS) mapping mechanisms for converged OFDMA-PON and LTE networks to satisfy the stringent QoS requirements. Various options for exhibiting QoS mapping between the LTE bearers and optical OFDMA-PON queues have been presented. These include, wireless classes forwarded directly to one priority optical queue (so called non-mapping mechanism), 1:1 mapping where class identifier, QoS channel indicator (QCI), levels are mapped to their respective priority optical queues and finally group mapping taking into account bandwidth availability at both enhanced node Bs (eNBs) and optical network units (ONUs) to accommodate optical and wireless user's requests. Performance figures, based on system level simulation, are drawn with respect to the QCI delay between eNBs and evolved packet core (EPC) for various numbers of users per cell.
\end{abstract}

Keywords: Optical and wireless convergence, OFDMA-PON, LTE, wireless backhauling, QoS provisioning

\section{INTRODUCTION}

With the advancements of next generation wireless networks, such as LTE [1], data rates across radio cells are expected to increase significantly [2]. The current infrastructure for backhauling this traffic from a base station to a central office, primarily based on microwave links, will not be sufficient for high data rates.

Next generation optical networks, such as OFDMA-PONs [3], could provide an alternative solution for high capacity backhauling links. In such converged scenarios an effective QoS mapping mechanism would be required between optical and wireless queues in order to avoid performance degradation of both types of users.

To that extent, this paper describes optimised OFDMA-PON layer-2 protocol and algorithms to accommodate LTE eNBs for QoS mapping. Performance evaluations was based on developing an event-driven packet-based, system level LTE simulator using OPNET. Various implementations of QoS mapping, namely non-mapping, 1:1 and group mapping have been developed. QCI latency and throughput performance figures have been drawn for increasing number of wireless users per cell.

\section{QoS MAPPING REQUIREMENTS FOR LTE BACKHAULING}

The QoS model of evolved packet system (EPS) for LTE, which was standardized in 3GPP release 8 [1], is based on the logical concept of an EPS bearer. Unlike LTE, typical PONs (including OFDMA ones as well) do not support bearer-based connections. Bandwidth requests are instead queue-oriented. An aggregate bandwidth is allocated from the optical line terminal (OLT) to each ONU which then makes a local decision to allocate the granted bandwidth and schedules packet transmission.

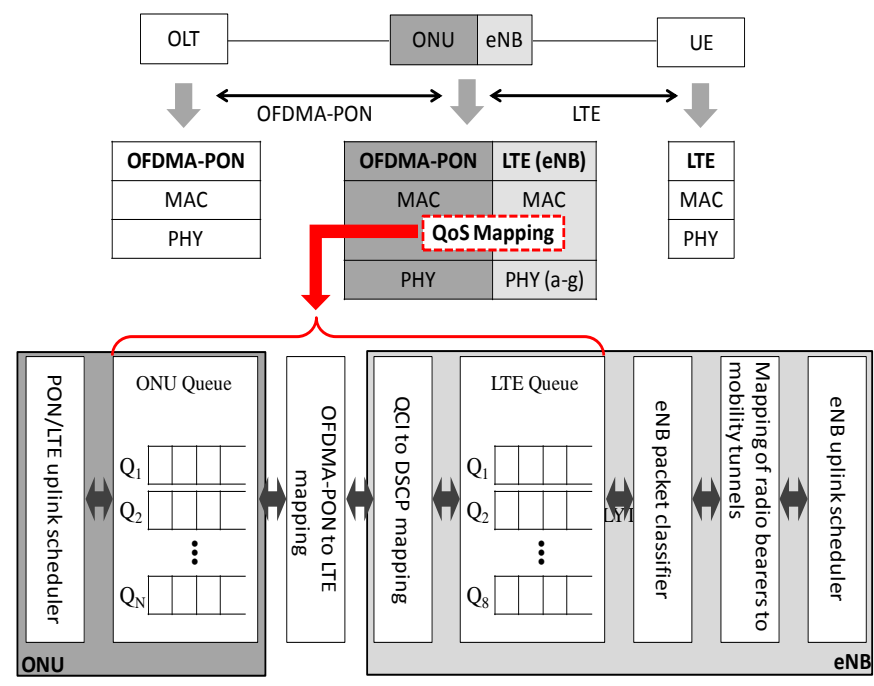

Figure 1. QoS mapping between LTE and the OFDMA-PON.

Therefore, to achieve a truly integrated scheduler, an effective mapping mechanism is required between the OFDMA-PON priority queues and the QCI/bearer-based LTE IP flows. In particular, as shown in Figure 1, 
mapping has to identify which LTE IP flow should be stored in which OFDMA-PON priority queue for the equivalent QoS. The figure also shows the internal data flow of ONUs and eNBs and the need for QoS mapping.

In terms of the number of QoS queues, the OFDMA-PON [4] accounts for three different priority queues in each ONU, while LTE defines eight standardized QCIs [1]. These classify data traffic into eight different classes of service, ranging from real-time gaming to the lowest priority best-effort TCP bulk data.

To enable a unified QoS mapping strategy between the PON and wireless services, will require the implementation of some critical functionalities. One would require that every ONU and OLT can be directly configured with the standardized QCIs of LTE (QoS characteristics) or more precisely with their corresponding diffServ code point (DSCP) values since the bearers are not visible to the OLT and ONUs.

In addition, the packet scheduler at ONUs and the OLT must apply the same packet forwarding methodology for both PON and wireless upstream/downstream traffic for each and every configured QCI/DSCP value that is associated with a given IP flow.

Another important aspect, associated closely with the bandwidth allocation, is that PONs use a cycle-based approach for DBA, indicating that bandwidth is allocated to each ONU for a specific time only. This could lead to unnecessary delays for the wireless users. The centralized OFDMA-PON architectures support differentiated upstream QoS via two independent scheduling mechanisms named inter- and intra-ONU. However, none of these scheduling mechanisms can guarantee bandwidth for real-time IP flows because the bandwidth allocated by the OLT to an ONU can only be guaranteed for a significantly short time (e.g. a fraction of one cycle) and may vary from one cycle to another according to the load among ONUs. Thus, each ONU is required to reserve bandwidth for its real-time IP flows for the whole duration of the flow (and not on a per cycle basis) in order to satisfy their QoS requirements as specified by the LTE DSCP value.

Taking the above into account, detailed mapping definitions and performance evaluation of the various scenarios is presented in the next section of this paper. An end-end data transfer has been achieved in the use of the OFDMA protocols and algorithms developed in $[4,5]$.

\section{EVALUATION OF THE DISTRIBUTION OF MAPPING WITHIN THE NETWORK}

\subsection{QoS mapping scenarios}

The performance evaluation was based in upstream, expanding on the developed QoS mapping mechanisms. A similar mapping distribution also applies in downstream. The two mapping methods developed include, 1:1 mapping and, what is defined as, group mapping. In view of the application of mapping, the performance of wireless users is investigated. A number of valid network scenarios have been simulated, distinguishing between the scheduling as well as the traffic conditions that influence bandwidth allocation.

Figure 2 (left) demonstrates 1:1 mapping where the same number of queues are available at both the eNB and ONU. This approach provides a direct scheduling mechanism since wireless data is stored, depending on bearer priority, to the equivalent priority optical queue. 1:1 mapping can apply to any number of queues, not only restricted to three. A potential limitation of 1:1 mapping could arise from the fact that the PON could have less queues available compared to LTE. As a result, full service consolidation might require the application of a more complicated mapping schedule. This has led to the introduction of group mapping.
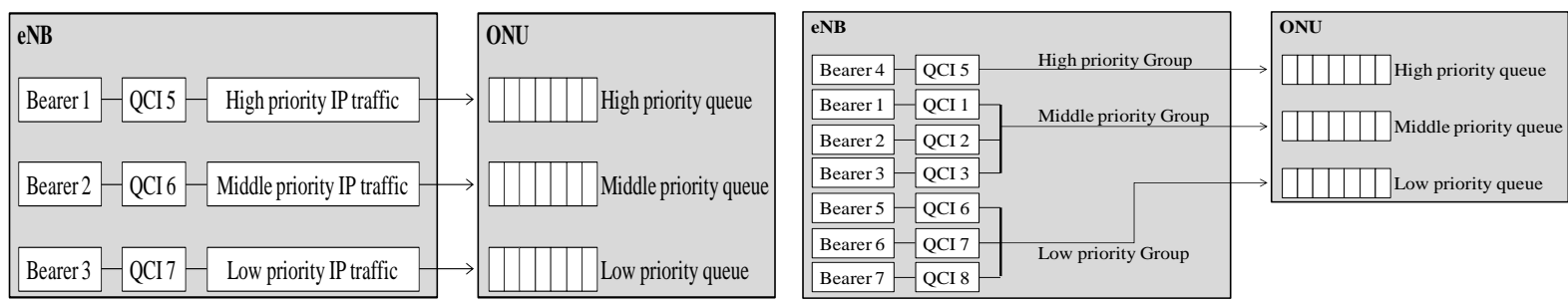

Figure 2. Example of 1:1 (left) and group (right) mapping between eNB and an ONU.

Group mapping assumes the number of wireless queues is greater than that of the ONU queues. For LTE mapping to a GPON-like OFDMA-PON the above assumption is directly true. A configuration based on 7 bearers (the LTE standard defines 8 bearers but the OPNET simulator only allows up to 7 bearers to be evaluated), consisting of 3 GBR (QCI1, 2 and 3) and 4 Non-GBR (QCI5, 6, 7 and 8) wireless services is presented in Figure 2 (right). The wireless groups are then mapped to their corresponding optical queues, as shown in the figure, in strict priority order. Group mapping assumes the same number of optical priorities as 1:1 mapping.

Both 1:1 and group mapping are finally contrasted to non-mapping, implemented by the 3 and 7 bearers respectively forwarded to a single optical queue, regardless of the priority of different QCIs. The wireless packets are thus transmitted on the PON in the same order they have been scheduled at the eNB. Non-mapping is expected to provide the baulk figures of end-end packet delay and converged network throughput, for wireless 
users, due to the introduction of the optical infrastructure. Also it presents a reference point for performance enhancement in view of mapping.

\subsection{Simulation scenarios}

Figure 3 provides a representation of the simulated network, distinguishing between the connection points of, residential (A) and wireless users (B) and the point in the process model where mapping takes place (C).

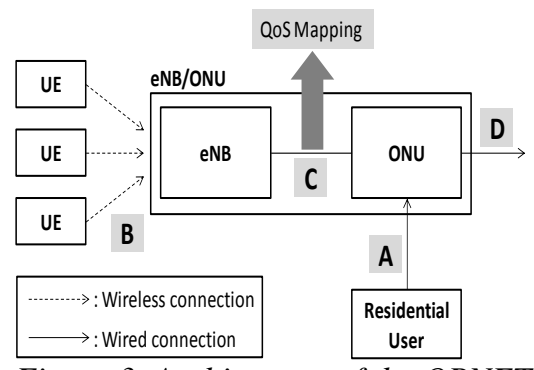

Figure 3. Architecture of the OPNET process model.
Table 1. Converged parameters

\begin{tabular}{|c|c|c|}
\hline Parameters & \multicolumn{2}{|c|}{ Description } \\
\hline Total network capacity upstream & \multicolumn{2}{|c|}{10 Gbps } \\
\hline Number of subcarriers & \multicolumn{2}{|c|}{64} \\
\hline Data rate per subcarriers & \multicolumn{2}{|c|}{$156.25 \mathrm{Mbps}(10 \mathrm{Gbps} / 64)$} \\
\hline \multirow{4}{*}{ Number of ONUs } & \multicolumn{2}{|c|}{32} \\
\hline & Residential ONU & eNB/ONU \\
\hline & 29 & 3 \\
\hline & \begin{tabular}{|l|} 
SLA0: SLA1: \\
SLA2 $=2: 10: 17$
\end{tabular} & SLA2 \\
\hline Distance between OLT and ONU & \multicolumn{2}{|c|}{$20 \mathrm{~km}$} \\
\hline Monitoring window time & \multicolumn{2}{|c|}{$2.0 \mathrm{~ms}$} \\
\hline Grant processing delay & \multicolumn{2}{|c|}{$5 \mu \mathrm{s}$} \\
\hline Propagation delay & \multicolumn{2}{|c|}{$5 \mu \mathrm{s} / \mathrm{km}$} \\
\hline $\begin{array}{c}\text { ONU offered load } 1.0 \\
\text { (Offered load for wired users) }\end{array}$ & \multicolumn{2}{|c|}{$312.5 \mathrm{Mbps}(10 \mathrm{Gbps} / 32)$} \\
\hline Packet size & \multicolumn{2}{|c|}{$\begin{array}{c}64-1518 \text { Bytes } \\
\text { (Uniformly generated) }\end{array}$} \\
\hline \multirow{3}{*}{$\begin{array}{l}\text { Traffic generation } \\
\text { for residential users }\end{array}$} & \multicolumn{2}{|c|}{ High prionity $\operatorname{CoS} 0: 20 \%$} \\
\hline & \multicolumn{2}{|c|}{ Middle prionity $\operatorname{CoS} 1: 40 \%$} \\
\hline & \multicolumn{2}{|l|}{ Low prionity } \\
\hline
\end{tabular}

Table 2. UE and eNB/ONU parameters

\begin{tabular}{|c|c|c|c|c|c|c|}
\hline \multicolumn{2}{|c|}{ Parameters } & \multicolumn{5}{|c|}{ Description } \\
\hline \multicolumn{2}{|c|}{ Inter site distance (ISD) } & \multicolumn{5}{|c|}{$3 \mathrm{~km}$} \\
\hline \multicolumn{2}{|c|}{$\begin{array}{l}\text { Modulation and coding scheme } \\
\text { index (MCS) }\end{array}$} & \multicolumn{5}{|c|}{22} \\
\hline \multicolumn{2}{|c|}{ HARQ } & \multicolumn{2}{|c|}{$\begin{array}{c}\text { Uplink } \\
\& \text { Downlink }\end{array}$} & \multicolumn{2}{|c|}{$\underset{\text { Max }}{\text { Metransmission }}$} & 3 \\
\hline \multicolumn{2}{|c|}{ Mobility of UE } & \multicolumn{5}{|c|}{$50 \mathrm{~km} /$ ( 1 sector per cell assumed) } \\
\hline \multicolumn{2}{|c|}{ Number of bearers per UE } & \multicolumn{5}{|c|}{3 and 7} \\
\hline \multirow{2}{*}{\multicolumn{2}{|c|}{ QCI of bearer }} & \multicolumn{2}{|c|}{3 Bearers } & \multicolumn{3}{|c|}{$5,6,7$} \\
\hline & & \multicolumn{2}{|c|}{7 Bearers } & \multicolumn{3}{|c|}{$1,2,3,5,6,7,8$} \\
\hline \multicolumn{2}{|c|}{ Data rate per UE (upstream) } & \multicolumn{5}{|c|}{$1.5 \mathrm{Mbps}$} \\
\hline \multirow{2}{*}{$\begin{array}{c}\text { Aggregate } \\
\text { wireless data rate } \\
\text { ("C" in Figure 3) }\end{array}$} & \begin{tabular}{|l|} 
Number of \\
UEs per cell \\
\end{tabular} & 10 & 20 & 30 & 40 & 50 \\
\hline & $\begin{array}{l}\text { Data rate } \\
\text { (Mbps) }\end{array}$ & 15 & 30 & 45 & 60 & 75 \\
\hline \multicolumn{2}{|c|}{$\begin{array}{l}\text { Residential data generation at } \\
\text { eNB/ONU } \\
(" A \text { " in Figure } 3)\end{array}$} & 235 & 220 & 215 & 200 & 200 \\
\hline \multicolumn{2}{|c|}{$\begin{array}{c}\text { Total data generation } \\
\text { (" } \mathrm{D} \text { " in Figure 3, Offered load of } \\
0.8)\end{array}$} & 250 & 250 & 250 & 250 & 250 \\
\hline
\end{tabular}

The simulation parameters required for the converged protocol evaluation in OPNET are summarized in Table 1. The bulk of parameters in Table 1 associated with the residential users, resemble those defined in [4]. These include the number of subcarriers and ONUs, the distribution of ONUs to SLAs, the traffic generation statistics and the distribution of residential users to service classes. The 29 residential ONUs and 3 hybrid eNB/ONUs are used to evaluate convergence. In addition to class of service (CoS) differentiation, the OFDMAPON layer-2 has traditionally also distinguished between service level agreements (SLAs) to define the various data rate levels applicable to different ONUs. Since the converged network emphasizes on mapping and how priority queues could be best utilized, only the low SLA, SLA2, is used for eNB/ONUs, to demonstrate the absolute worst-case scenario.

Finally, Table 2 summarises the LTE and eNB/ONU parameters, needed to be specified for the simulations.

\section{PERFORMANCE EVALUATION}

This section draws the QCI packet delay characteristics obtained for different LTE bearers over OFDMA-PON with respect to increasing user equipment (UE) numbers per cell. QoS mapping was evaluated by contrasting 1:1 mapping ( 3 bearers to $3 \mathrm{CoS}$ ) and group mapping ( 7 bearers to $3 \mathrm{CoS}$ ) against non-mapping, for the reasons described in previous sections, implemented by 3 bearers and 7 bearers respectively forwarded to a single optical queue. A fixed eNB/ONU load of 0.8 was assumed.

Figure 4 (left) displays the wireless packet delay, measured with non-mapping (three bearers forwarded to the low priority ONU queue) and in the application of 1:1 mapping, with residential users present at the network
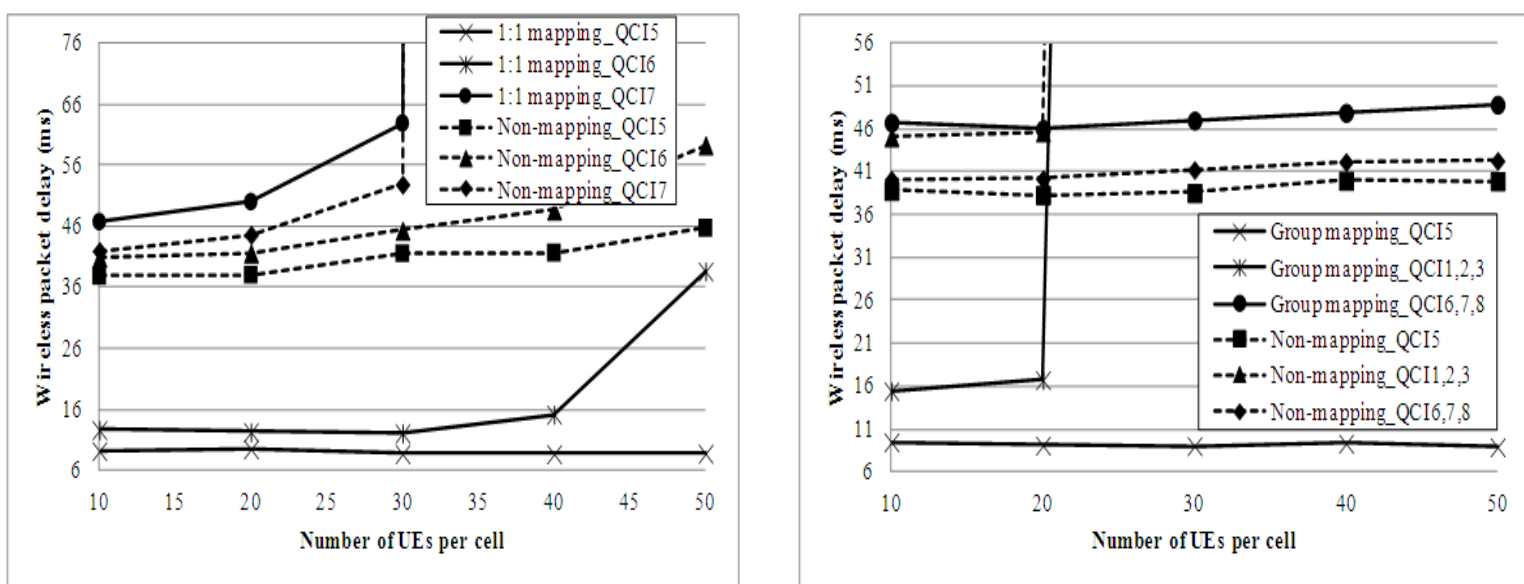

Figure 4. Packet delay for 1:1 (left) and group mapping (right) compared to non-mapping mechanism.

ONU. When the eNB/ONU employs 1:1 QoS mapping, the high priority bearer (carrier of QCI 5) and middle priority bearer (QCI 6) display notably decreased packet delay compared to non-mapping. A clear characteristic with almost a fourfold reduction in delay is demonstrated for the highest priority bearer (QCI 5) at 50 UEs per cell (from 11 to $46 \mathrm{~ms}$ ). Correspondingly, a threefold reduction in delay is also presented for the middle priority 
bearer (QCI 6) at 30 UEs per cell (14 ms to $46 \mathrm{~ms}$ ). The steep delay responses generated at 30 UEs for the low priority bearer (QCI 7) is due to the bottleneck between UEs and the eNB at increased UE numbers in a cell, with or without QoS mapping.

In contrast, non-mapping presents a slight, by 1.2 times, reduction in packet delay for the lowest bearer (QCI 7) at 30 UEs, compared to its mapping counterpart (53 to $63 \mathrm{~ms}$ ). The reason for this abnormality is justifiable if the scheduling methodology between mapping and non-mapping is carefully considered. In mapping, upon receiving the Grant messages from the OLT, the eNB/ONU transmits data first from the high priority queue, then from the second highest priority and last from the low priority queue. Thus the lowest priority queue is relatively disadvantaged. Instead, in the absence of mapping, the wireless data can be disadvantaged with respect to the higher, residential data rates, leading to higher packet delays. Due to the fact in non-mapping all LTE queues are forwarded to the low priority optical queue, the packet delay between bearers is not much different from one another, ranging as being confirmed by Figure 4 (left) from $37 \mathrm{~ms}$ to about $56 \mathrm{~ms}$.

Figure 4 (right) presents group mapping, contrasted again to non-mapping, implemented by the transition of the 7 wireless bearers to the low priority ONU queue. The QCI5 bearer emulates high priority, while QCI1, 2 and 3 and QCI6, 7 and 8 bearer groups signify respectively, middle and low priorities [1]. Unlike the results observed earlier, following the simulation of 3 bearers, the performance of the middle priority group (QCI1, 2 and 3) in Figure 4 (right) saturates early at 30 UEs. This can be pinned down to the use of GBR services, comprising, in the 7 bearers mapping, the middle priority group. UEs operating GBR services require admission control from the eNB for their activation. If failed to be activated, GBR data is transmitted in the default bearer. Following the simulation specifications, the eNB allows only four GBRs per cell. Thus the remaining GBRs are communicated in the default bearer.

Finally, the packet delay of the low priority group is $6 \mathrm{~ms}$ higher with group mapping, compared to nonmapping, because of the strict priority followed with intra scheduling.

\section{CONCLUSIONS}

In a converged optical and wireless network the QoS mapping between the base stations and ONUs is critical in optimising the performance of both the optical and wireless users. The QoS mapping scenarios and performance evaluation presented in this paper were based on LTE system-level investigations over the next generation OFDMA-PON. The results obtained focused on QCI delay versus the aggregate wireless data rate upstream for dynamic bandwidth allocation. When the eNB/ONU employs 1:1 QoS mapping, the high priority bearer (carrier of QCI 5) and middle priority bearer (QCI 6) display notably decreased packet delay compared to non-mapping. If group mapping is employed instead (considering 7 bearers thus more realistic scenario) the performance of the middle priority group (QCI1, 2 and 3) saturates early, at 30 UEs. This can be pinned down to the use of GBR services, comprising, in the 7 bearers mapping, the middle priority group. Significantly, the obtained delays for all mapping mechanisms are well within the limits defined by the LTE standard demonstrating an efficient high capacity backhauling link for next generation wireless access.

\section{ACKNOWLEDGEMENTS}

Aspects of this work have been developed to support the European FP7 ACCORDANCE project.

\section{REFERENCES}

[1] D. Astely, E. Dahlman, A. Furuskar, Y. Jading, M. Lindstrom, and S. Parkvall: LTE: the evolution of mobile broadband - LTE part II: 3GPP release 8, Communications Magaz., vol. 47, pp. 44-51, Apr. 2009.

[2] P. Bhat, S. Nagata, L. Campoy, I. Berberana, T. Derham, L. Guangyi, S. Xiaodong, Z. Pingping, Y. Jin: LTE-advanced: an operator perspective, Communications Magaz., vol. 50, pp. 104-114, Feb. 2012.

[3] K. Kanonakis, I. Tomkos, H. G. Krimmel, F. Schaich, C. Lange, E. Weis, J. Leuthold, M. Winter, S. Romero, P. Kourtessis, M. Milosavljevic, I. Cano and J. Prat: An OFDMA-Based Optical Access Network Architecture Exhibiting Ultra-High Capacity and Wireline-Wireless Convergence, Communications Magaz., 2012 (to be published).

[4] ACCORDANCE. (2012, April 20 $0^{\text {th }}$ ). Definition and evaluation of algorithms for dynamic bandwidth allocation in ACCORDANCE (D4.3) [Online]. Available: http://ict-accordance.eu/.

[5] W. Lim, P. Kourtessis, M. Milosavljevic and J.M. Senior: QoS aware MAC protocol for OFDMA-PON, in Proc. ICTON 2011, Stockholm, Sweden, June 2011. 